\title{
Stadtkonzepte als aktuelle Perspektiven auf die Stadt
}

Rezension zu Dieter Rink / Annegret Haase (Hg.) (2018): Handbuch Stadtkonzepte. Analysen, Diagnosen, Kritiken und Visionen. Stuttgart: Barbara Budrich.

Abb. 1 Titel des Buches (Quelle: Barbara Budrich Verlag)

Handbuch Stadtkonzepte

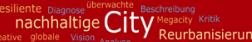

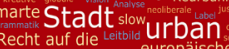
cosolisticteuropa
Der urbane Raum hat sich längst nicht nur zu einem spezifischen gesellschaftlichen Format, sondern auch zu einer alles dominierenden gesellschaftlichen Konstruktion von Wirklichkeit, ja einer alles dominierenden Lebensform, zu einem alles beherrschenden globalen Habitus entwickelt. Unzählige Beschreibungen, Diagnosen und Prognosen sind in diesem Zusammenhang entstanden und weltweit zur Basis von Leitbildern, Bauprojekten und $\mathrm{Zu}$ kunftskonzepten gemacht worden. Gleichzeitig gerät aber gerade auch diese Lebensform immer wieder in die Kritik, da sie zunehmend Konflikte und Krisen hervorruft und das Zusammenleben immer problematischer erscheinen lässt. So ist inzwischen auch schon von einer ,city of crisis' die Rede. Genau diese einerseits hochattraktive und anderseits offenbar gleichzeitig von vielfältigen sozialen, ökonomischen und religiösen Spannungen geprägte paradoxe Konstellation bildet den Hintergrund, vor dem sich die Stadtökologin Annegret Haase und der Stadtsoziologe Dieter Rink auf die Suche nach tragfähigen ,Stadtkonzepten', wie sie ausdrücklich formulieren, machen. Sie betrachten dabei freilich jene teilweise sogar widersprüchlichen, mal geradezu von Euphorie, mal von extremer Skepsis geprägten Einschätzungen urbaner Lebensformen vorwiegend als bloße Effekte unterschiedlich ausgerichteter Perspektiven. Diese miteinander quasi konkurrierenden Perspektiven führen zu entsprechend verschieden ausgerichteten Diagnosen und Prognosen, Leitbildern und Programmatiken. So gesehen liegt es durchaus nahe, diese unterschiedlichen Blickwinkel städtebaulicher, künstlerischer, wissenschaftlicher, politischer und publizistischer Provenienz erst einmal einzeln zu würdigen, dabei aber so vorzugehen, dass sie sich miteinander vergleichen lassen, sodass man daraus gegebenenfalls Folgerungen für die Zukunft der Stadt entwickeln kann. Auch angesichts der hiesigen Debatte über teils zunehmend attraktive und teils immer desolatere, schrumpfende Städte und die damit verknüpften vielfältigen Herausforderungen scheint dies zumindest auf den ersten Blick nicht nur ein spannendes, sondern vor allem auch ein sehr nützliches Vorhaben zu sein.

In dem Sammelband werden 21 Stadtkonzepte verschiedener Autor_innen dargestellt. Dabei werden noch nicht einmal alle Ansätze einbezogen, 
die derzeit in der Diskussion sind, sondern nur solche, die für die konkrete Stadtentwicklungssituation relevant erscheinen. Es wird also weniger auf globale Konzepte wie zum Beispiel die ,New Urban Agenda' gesetzt, obwohl sich dieses Konzept seit HABITAT I von 1976 breit entwickelt hat und es zuletzt 2016 auf der UNO-Konferenz reformuliert worden ist. Genauso werden solche Konzepte beiseitegelassen, die nur planerische Aspekte betreffen oder entweder rein retrospektiv oder rein utopisch ausgerichtet sind.

Stattdessen werden diejenigen Ansätze in den Mittelpunkt gerückt, die konkret Eingang in die hiesigen alltags- und stadtpolitischen Debatten gefunden haben, sich also, wenn auch in sehr unterschiedlicher Weise, direkt auf die hiesige urbane Entwicklung beziehen. Solche Konzepte werden hier ausdrücklich als ,Stadtkonzepte bezeichnet. Es werden also ganz bewusst gerade solche Konzepte ausgewählt, die im Umfeld der Stadtdebatten zurzeit diskutiert werden und die sich infolgedessen auch gut eingrenzen, abgrenzen, in ihrer Reichweite einschätzen und diskursiv verorten lassen, und die mithin eine vergleichbare und einschätzbare Komplexität und Relevanz aufweisen. Solche eher praktisch ausgerichteten Stadtkonzepte von der ,globalen Stadt über die ,grüne Stadt‘ bis zur ,nachhaltigen Stadt' und zur ,postsäkularen“ und ,postsozialistischen Stadt' sollen mit ihren jeweils prägenden Analysen, Diagnosen und Trends schrittweise ins Blickfeld gerückt und die damit verknüpften planerischen bis politischen Diskurse, deren jeweilige Ziele und Vermarktungsweisen, deren Leitbild- und Steuerungsfunktion herausgearbeitet und auf diese Weise vergleichbar gemacht werden. Im Einzelfall werden zum besseren Verständnis eines Konzeptes auch gesellschaftstheoretische Hintergründe mit einbezogen. Ansonsten werden die jeweiligen Konzepte bewusst immanent, in ihrer spezifischen Vielschichtigkeit und ihren Entwicklungspotentialen in ihrem jeweiligen Entstehungszusammenhang und ihrem speziellen Anliegen entsprechend gewürdigt.

Die Vorgehensweise wird bei der Darstellung solcher Konzepte wie,Nachhaltige Stadt' oder ,Smart City' besonders deutlich. Das Konzept wird jeweils zunächst aus seinem Diskurszusammenhang heraus analysiert und ins Blickfeld gerückt. Bei der weiteren Vorstellung der jeweiligen Stadtkonzepte wird immer nach den gleichen Schritten vorgegangen. Diese Schritte werden schon in der Einleitung detailliert dargestellt und sind insoweit auch sehr überzeugend. Allerdings impliziert diese Fokussierung auf für alle Analysen gleichermaßen verbindliche Schritte und die Abgrenzung von positionellen Sichtweisen auch, dass die Stadtkonzepte im Prinzip nur formal analysiert und damit auch nur formal vergleichbar gemacht werden. Es wird kein systematischer Bezug zu einem möglichen tertium comperationis hergestellt, also zu einem gemeinsamen Referenzrahmen, in diesem Fall zu einer Vorstellung von dem, was eine Stadt als spezifische Form des Zusammenlebens, als eine Stadtgesellschaft im Kern ausmacht. Stattdessen gehen Haase und Rink in ihrem Handbuch davon aus, dass das, was eine Stadt ausmacht, letztlich aus der Summe dessen abgeleitet werden kann, was an Konzepten in der Stadtforschung und in der einschlägigen angewandten Forschung bis hin zu den Planungswissenschaften und dem Städtebau angesagt ist.

Die Vorstellung, dass am Ende aus dem, was sich aus den Vergleichen unter dem Strich ergibt, eine aussagekräftige Zusammenschau dessen herauskristallisiert, was eine Stadt heute darstellt, wird in der abschließenden 
Reflexion freilich deutlich relativiert. Hier wird nämlich die Erwartung formuliert, dass das Handbuch im Rahmen der dargestellten Vielfalt und Komplexität die Chance eröffnet, gegebenenfalls auch ein neues Verständnis über urbane Wirklichkeit zu ermöglichen. Letztes Ziel besteht eben darin, sich Klarheit darüber zu verschaffen, in welcher Stadt wir zukünftig leben ,wollen'. Insofern ist die Absicht, einfach alles bloß zusammen zu rechnen, eine eher vorläufige Positionierung. Und tatsächlich wird diese Positionierung auch in vielen Beiträgen deutlich überschritten. Jedenfalls wird vielfach, so zum Beispiel bei Janet Merkel (207), gefordert, stärker von einer integrierten Stadtentwicklung auszugehen.

Schon der erste Beitrag von Frank Eckardt über „Austerity Urbanism“ ist sehr spannend. Hier wird nicht nur deutlich gemacht, welche Linie im Rahmen dieses Konzepts entwickelt wird, sondern auch, dass dieses Konzept eigentümlich normativ ausgerichtet einem abstrakten Sparprinzip folgt und dabei ganz gezielt auf Verarmung sowohl privater als auch öffentlicher Haushalte setzt und gleichzeitig die fundamentalen Aufgaben und Aufgabenzuteilungen im staatlichen wie kommunalen Handeln, einfach alle lokalen Bedürfnisse und Erfordernisse, bewusst ausblendet (38f.). Mit diesem Konzept werden also weniger vorgegebene urbane Gegebenheiten weiterentwickelt, als vielmehr quasi stadtkontextfreie politische Prioritäten postuliert und der urbanen Wirklichkeit quasi aufgezwungen. Es werden dabei zum Beispiel die vielfältigen Faktoren von kommunaler Verschuldung verkannt. Eckardt plädiert abschließend dafür, unter der Stadtbevölkerung einen Verständigungsprozess über kommunale Aufgaben und Ausgaben in Gang zu bringen. Seine Einschätzung und die Folgerungen sind sehr überzeugend. Solche Überlegungen sind freilich nur möglich, wenn man sich nicht auf eine rein immanente Darstellung des Konzeptes beschränkt, sondern dieses auch daraufhin überprüft, was es für ein urbanes Zusammenleben bedeutet. Schon in dieser Darstellung wird also durchaus begründet der für die Einzelanalyse gesteckte Rahmen erfolgreich überschritten.

Im Beitrag von Nina Schuster über „Diverse City“ wird ein gleichsam umgekehrt ausgerichtetes Konzept dargestellt. Hier geht es nicht um die Durchsetzung eines normativen Impulses, sondern um die sozial adäquate Erfassung einer zwar hoch umstrittenen und immer wieder verdrängten urbanen Grundeigenschaft, die allerdings eigentlich eine urbane Selbstverständlichkeit ist, nämlich Vielfalt. Dieser Vielfalt wird häufig sogar ein hohes gesellschaftliches Potential zugesprochen. Schuster plädiert dafür, dieses Potential entschlossener als bisher aufzugreifen. In diesem Zusammenhang geht sie zum Beispiel auf die, sanctuary cities' ein, die Vielfalt eben auch durch das Engagement für Migration gesellschaftspolitisch hervorheben wollen. Sie verweist aber auch auf das Problem der strukturellen Einschränkungen von Vielfalt durch Segregation, Benachteiligung, Verengung auf soziale, ethnische oder nationale Zugehörigkeiten. Hier wäre eine neue Wertschätzung von Vielfalt ein erster Schritt in die richtige Richtung. Obwohl dieses Konzept völlig anders als das erste Konzept aufgebaut ist, wird es auch hier erst spannend, wo Vorstellungen davon durchklingen, was urbanes Zusammenleben eigentlich generell ausmacht.

Ganz ähnlich gelagert ist das, was Sandra Huning über „Feminismus und Stadt" notiert. Auch in diesem Fall wird die Relevanz von Gender für das 
Zusammenleben, für die Architektur und Stadtplanung eingeklagt. Auch in diesem Fall gilt es, eine Grundeigenschaft des urbanen Zusammenlebens in den Blick zu nehmen (112f.). Und analog geht es darum, das Zusammenleben aus feministischer Perspektive heraus immer wieder neu auszurichten. Letztlich ist es die Intersektionalitätsdebatte, die die Genderthematik und die Diversity-Thematik im Blick auf das urbane Zusammenleben miteinander verknüpft.

Ein weiterer Konzepttypus wird deutlich, wo von Rink die „Nachhaltige Stadt" vorgestellt wird. Hier geht es weder um eine abstrakte politische Normsetzung noch um ein den Kern des urbanen Zusammenlebens schon immer betreffendes, nur bislang gegebenenfalls zu wenig gewürdigtes Phänomen, sondern es geht um eine neuartige globalgesellschaftliche Herausforderung, die eben auch vor der Stadtgesellschaft nicht Halt macht, ja gerade hier besonders virulent wird, weil die Städte als die längst dominierende Lebensform im Rahmen der globalen Umweltproblematik eine zentrale Rolle spielen. Diese Herausforderung hat offenbar innerhalb der Stadtentwicklung zunächst zu eher normativen Aktionen, später zu Chartakonzepten und schließlich auch zu ganz praktischen Transfermodellen geführt. So hat sich schrittweise ein neuartiges Stadtkonzept herausgebildet. Es ist so etwas wie ein Impulskonzept, also ein Konzept, das dazu auffordert, fortschreitend Einsichten im Kontext globaler Herausforderungen zu gewinnen und sie dann auf lokaler Ebene zu reformulieren, um daraus schließlich entsprechende Folgerungen zu ziehen.

Dieser Konzepttypus lässt sich auch in dem Beitrag von Haase über die „Grüne Stadt“ erkennen, in dem am Ende ganz konkrete Schritte diskutiert werden. Ähnlich sieht es beispielsweise auch in dem Beitrag von Sigrun Kabisch und Frauke Kraas über die „Megastadt“ aus. Die im Rahmen des globalen Wandels entstandenen Megastädte und die in diesem Zusammenhang entstandenen besonderen Herausforderungen für ein megastädtisches $\mathrm{Zu}$ sammenleben mit großen informellen Zonen, migrations- beziehungsweise ankunftsgeprägten Vierteln und so weiter evozieren Zerfalls- und Fragmentierungstendenzen, die in einem ganz besonderen $\mathrm{Ma} \beta$,guter Regierungsführung' und ,nachhaltig ausgerichteter Entwicklungspfade' bedürfen. Auch kommt es zu Impulskonzepten, die in diesem Fall auf ganze, urbane Korridore abzielen. Das Ergebnis sind Stadtkonzeptionen, die hochdynamische globale Mobilitätseffekte und damit die Sicherung der Lebensbedürfnisse zunehmend extrem wachsender Bevölkerungsgruppen in einem urbanen Raum in den Mittelpunkt rücken. Solche Konzeptionen bleiben freilich angesichts der hier beobachtbaren extremen Komplexität auch in ihrem Impulscharakter nur modellhaft.

Schließlich werden eine ganze Reihe von Stadtkonzepten vorgestellt, die gar nicht auf die Stadt insgesamt abzielen, sondern auf einzelne Aspekte des urbanen Zusammenlebens. Hier werden einzelne Themen des Zusammenlebens aufgegriffen, und deren Bedeutung für die Stadtentwicklung lokalisiert, eingegrenzt und gewürdigt.

Besonders gut gelingt das Andrej Holm mit seinem Beitrag über das „Recht auf Stadt“. Ihm geht es um die Entwicklung von Vorstellungen über das, was die individuelle Position in einer urbanen Gesellschaft ausmacht, um deren zivilgesellschaftliche Präsentation und um die diskursive Durchsetzung und Umsetzung damit verknüpfter Anliegen und 
Erwartungen. Dabei entstehen Fragen im Hinblick auf die Gleichstellung des Einzelnen, eine Umverteilung von Ressourcen, auf Inklusion bis hin zu Antigentrifizierungsmaßnahmen. Hier wird auf anthropogene Erfordernisse Bezug genommen, zum Beispiel das Recht auf eine Wiederaneignung des städtischen Raumes und der lokalen Ressourcen.

Durchaus vergleichbar sind Konzepte wie die „Resiliente Stadt“ ausgerichtet. Christian Kuhlicke skizziert hier gleich fünf unterschiedliche Versionen dieses Konzeptes, die zum Teil eher vage mit dem Resilienzmoment argumentieren, zum Teil aber auch darauf aus sind, ganz klar alternative Stadtentwicklungspotentiale zu fördern. Auch hier geht es um eine Rekonstruktion, wie sich Vorstellungen entwickeln, wie sie sich im urbanen Alltag verankern und wie sie zu einem kollektiven Handlungspotential avancieren. Am Anfang stehen dabei nicht theoretische Vorstellungen über das, was eine urbane Gesellschaft ausmachen mag, sondern ganz alltagspraktisch erlebte Irritationen und Verunsicherungen. Im Rahmen einer solchen Erfahrung kristallisieren sich Positionierungen heraus, die unterhalb der urbanen Institutionen lebendig werden, insofern alternativ erscheinen und zu einem resilienten Potential anwachsen können. Die Herausforderung für eine Stadt besteht dann darin, hier eine angemessene Responsibilität zu organisieren statt auf Entpolitisierung oder Konfliktnaturalisierung zu setzen. Hier ist die lokale Öffentlichkeit gefragt.

Auch solche Überlegungen wie die von Katrin Großmann zur „Just City“ gehören in diesen Zusammenhang. Unter diesem Label summiert sie die Diskussionen über Verteilungsgerechtigkeit, aber auch über Differenz- und Prozessgerechtigkeit. Hier geht es immer wieder um Prinzipien einer inklusiven, alle beteiligenden, partizipativ ausgerichteten Stadtentwicklung. Der Beitrag ist deshalb besonders aktuell, weil er die Gerechtigkeitsfrage gerade auch mit Blick auf den Wohnungsmarkt diskutiert. Es geht auch hier eigentlich um alte Fragen, die in der aktuellen Entwicklung neue Brisanz gewonnen haben. In diesen Zusammenhang gehören auch Konzepte wie das von Jan Wehrheim dargestellte Konzept „Überwachte Stadt“.

Tatsächlich werden in dem Sammelband eine ganze Fülle von Stadtkonzepten dargestellt, die hier gar nicht alle aufgegriffen werden konnten. Deutlich werden sollte, dass es in der Tat gelungen ist, wichtige Elemente urbaner Entwicklung zu markieren und in ihrer Bedeutung klar zu akzentuieren. Dabei ist auch eine ganze Reihe offenbar grundsätzlicher Probleme sichtbar geworden. Insbesondere wird immer wieder die Partizipation der Bevölkerung angemahnt. Was hier fehlt, ist der Versuch, vor dem Hintergrund dieser vielfältigen Konzepte einen Referenzrahmen zu skizzieren, der nicht nur als tertium comparationis, sondern auch als Basis für eine die gesellschaftliche Konstruktion der urbanen Wirklichkeit beziehungsweise den urbanen Raum in den Blick nehmende Stadtentwicklungsdebatte dienen könnte. Eine diesbezügliche Zusammenführung der in diesem Sammelband präsentierten Segmente steht noch aus. In der abschließenden Auswertung klingt dies freilich durchaus an. Das würde auch eine solche Debatte wie die im Beitrag von Stefan Siedentop skizzierte über „Reurbanisierung“ oder die von Florian Koch über die „Europäische Stadt“ beflügeln. Die Zusammenführung der Beiträge zu einer solchen Vision sollte man nicht dem Leser oder der Leserin allein überlassen. 


\section{Autor_innen}

Wolf-Dietrich Bukow ist Soziologe mit den Schwerpunkten Migration, Mobilität, Diversität, Quartierforschung und Stadtentwicklung.

wbukow@uni-koeln.de

\section{Literatur}

Eckardt, Frank (2018): Austerity Urbanism. In: Rink, Dieter / Haase, Annegret (Hg.), Handbuch Stadtkonzepte. Analysen, Diagnosen, Kritiken und Visionen. Stuttgart: Barbara Budrich, 23-42.

Großmann, Katrin (2018): Just City. In: Rink, Dieter / Haase, Annegret (Hg.), Handbuch Stadtkonzepte. Analysen, Diagnosen, Kritiken und Visionen. Stuttgart: Barbara Budrich, 169-192.

Haase, Dagmar (2018): Grüne Stadt. In: Rink, Dieter / Haase, Annegret (Hg.), Handbuch Stadtkonzepte. Analysen, Diagnosen, Kritiken und Visionen. Stuttgart: Barbara Budrich, 151-168.

Holm, Andrej (2018): Recht auf Stadt. In: Rink, Dieter / Haase, Annegret (Hg.), Handbuch Stadtkonzepte. Analysen, Diagnosen, Kritiken und Visionen. Stuttgart: Barbara Budrich, 339-358.

Huning, Sandra (2018): Feminismus und Stadt. In: Rink, Dieter / Haase, Annegret (Hg.), Handbuch Stadtkonzepte. Analysen, Diagnosen, Kritiken und Visionen. Stuttgart: Barbara Budrich, 107-128.

Kabisch, Sigrun / Kraas, Frauke (2018): Megastadt. In: Rink, Dieter / Haase, Annegret (Hg.), Handbuch Stadtkonzepte. Analysen, Diagnosen, Kritiken und Visionen. Stuttgart: Barbara Budrich, 213-236.

Koch, Florian (2018): Europäische Stadt. In: Rink, Dieter / Haase, Annegret(Hg.), Handbuch Stadtkonzepte. Analysen, Diagnosen, Kritiken und Visionen. Stuttgart: Barbara Budrich, 87-106.

Kuhlicke, Christian (2018): Resiliente Stadt. In: Rink, Dieter / Haase, Annegret (Hg.), Handbuch Stadtkonzepte. Analysen, Diagnosen, Kritiken und Visionen. Stuttgart: Barbara Budrich, 359-380.

Merkel, Janet (2018): Kreative Stadt. In: Rink, Dieter / Haase, Annegret (Hg.), Handbuch Stadtkonzepte. Analysen, Diagnosen, Kritiken und Visionen. Stuttgart: Barbara Budrich, 193-212.

Rink, Dieter (2018): Nachhaltige Stadt. In: Rink, Dieter / Haase, Annegret (Hg.): Handbuch Stadtkonzepte. Analysen, Diagnosen, Kritiken und Visionen. Stuttgart: Barbara Budrich, 237-258.

Rink, Dieter / Haase, Annegret (Hg.) (2018): Handbuch Stadtkonzepte. Analysen, Diagnosen, Kritiken und Visionen. Stuttgart: Barbara Budrich.

Schuster, Nina (2018): Diverse City. In: Rink, Dieter / Haase, Annegret (Hg.), Handbuch Stadtkonzepte. Analysen, Diagnosen, Kritiken und Visionen. Stuttgart: Barbara Budrich, 63-86.

Siedentop, Frank (2018): Reurbanisierung. In: Rink, Dieter / Haase, Annegret (Hg.), Handbuch Stadtkonzepte. Analysen, Diagnosen, Kritiken und Visionen. Stuttgart: Barbara Budrich, 381-404.

Wehrheim, Jan (2018): Überwachte Stadt. In: Rink, Dieter / Haase, Annegret (Hg.), Handbuch Stadtkonzepte. Analysen, Diagnosen, Kritiken und Visionen. Stuttgart: Barbara Budrich, 451-472. 